Gastroenterologia 1967;107(Suppl. 1):1-5

\title{
Contents, Vol. 107, 1967
}

Verhandlungen der Deutschen Gesellschaft für Verdauungs- und Stoffwechselkrankheiten XXIII. Tagung in Wien 23.-25. September 1965

Im Auftrage des Vorstandes herausgegeben vom ständigeo Schriftführer der Gesellschaft Prof. Dr. G. A. MARTINI

Marburg/Lahn

Mit 37 Abbildungen

\section{BASEL (Schweiz) \\ S. KARGER NEW YORK}

Supplementum ad Vol. 107

GASTROENTEROLOGIA

International Review of Gastroenterology

Internationale Zeitschrift für Gastroenterologie

Revue Internationale de Gastro-entérologie

Redactorest H. BART£LH£IMER - M. DEMOLE - Th. C. HUNT - B. IHRE Curatore $\beta i$ L.

DEMLING - H. KAPP

Bedeutung der Zahlen am Kopf der Seiten:

Die Zahlen am äusseren Rand der Seiten bezeichnen die Seiten des Supplementurns zu

Gastroenterologia, diejenigen am inneren Rand die Seiten des Berichtes über die 23. Tagung der

Đeutschen Geßell $\beta$ chaft für Verdauungs- und Stoffwechselkrankheiten.

Meaning of numbers at the top pages:

The numbers on the outer side of the pages refer to the regular pages of the Supplementum to Gastroenterologia, and those on the inner side to the Proceeding $\beta$ of the 23rd Meeting of the Deutsche Gesellschaft für Verdauung $\beta$ - und Stoffwechselkrankheiten.

Signification des chiffres au haut des pages:

Les numé ro $\beta$ à Cangle exteme dee pages se rapportent aux pages du Supplementum à Gastroenterologia,

ceux à Tangle interne aux pages des comptes rendus de la 23e Reunion de la Deutsche Gesellschaft für

Verdauungs- und Stoffwechselkrankheiten.

AHe Rechte, insbesondere das der Übersetzung in fremde Sprachen, vorbehalten. Ohne ausdrückliche Genehmigung des Verlages i $\beta$ t es auch nicht gestattet, dieses Buch oder Teile daraus auf photomechanischem Wege (Photokopie, Mikrokopie) zu vervî́elíaltigen.

(C)

Copyright 1967 by S. KaГger AG, Basel Printed in Switzerland by Buchdmckerei Werner \& Bischoff AG, Basel Cliches: Aberegg-Steiner \& Cíe. AG, Bern, und Steiner \& Cie. AG, Basel Inhaltsverzeichnis

1. Hauptthema: Der operíerte Magen 
Meythaler, F. (Erlangen): Kohlenhydratstoffwechselstörungen bei Magen- und Darmerkrankungen (zugleich ein Beitrag zur Pathogenese des Dumping-Syndroms) (214) 6

Schultís, K.; Wagner, E. und Sailer, F. X. (Giessen): Die Resorption von D-Xylose vor und nach Magenresektionen (218) 10 Kuhlencordt, F. und Bartelheimer, H. (Hamburg): Die Auswirkungen der Magenresektion auf das Skelett (222) 14

Berndt, H. (Berlin): Spätfolgen der totalen Gastrektomie ～(227) 19 Bartsch, W. M. (Bonn): Proteolyse und Serumeiweiss nach totaler Magenresektion (231) 23

Luchmann, A. (Bad Salzig): Zur Cholelithiasis bei Magenresektion nach Billroth II (234) 26

Chlebowskí, J. und Dubowícka-Gabryelewíczowa, M. (Bialystok):

Eigene Beobachtungen über den Postgastroektomiesyndrom .... (237) 29

Iliescu, G.; Mínculescu, D.; Popa, G.; Splouchal, J.; Iancu, N. und Enescu, G. (Bukarest): Der vergleichende Wert der Vagotomie und der «Reconversions-Operation» nach Soupault-Bucaille im Dumping-Syndrom (240) 32

Iliescu, G.; Splouchal, J.; Popa, G. und Enescu, G. (Bukarest): Eine neue Technik der totalen Magenplastik (243) 35

Merkel, K. L. (Weyer): Lungentuberkulose und Magenresektion .... (248) 40

Partílla, H. (Wien): Aminosäurebestimmung im Stuhl bei normalen und resezierten Mägen (250) 42

2. Hauptthema: Gastroskopísche Diagnostik

Henníng, N. (Erlangen): Fortschritte der Gastroskopie, Biopsie und Zytologie in der Magendiagnostik (253) 45

Ofner, H. (Wolfsberg): Gastroduodenoskopie und farbphotographische Dokumentation mit dem Fiberscope (259) 51

Affolter, H. und Kapp, H. (Basel): Zur diagnostischen Bewertung der Gastroskopie (263) 55

Mahlo, A. (Hamburg): Die Saugbiopsie des Kardiagebietes (267) 59

3. Hauptthema: Postcholecystektomíesyndrom

Franken, F. H. (Düsseldorf): Klinik und Therapie der Gallensteinerkrankung in den letzten Jahrhunderten (271) 63

Koelsch, K. A. (Magdeburg): Beschwerden, Befunde, Diagnosen nach Cholecystektomie (275) 67

4 Suppl.

Inhaltsverzeichnis

Tagung 212

Mallet-Guy, P. (Lyon): Über die Wirklichkeit des sogenannten PostCholecystektomiesyndroms (279) 71

Nietlispach, L.; Marzoli, G. P. und Födísch, F. J. (Aarau): Morphologische Grundlagen des Postcholecystectomie-Syndroms $\quad$ (282) 74 Wewalka, F. (Wien): Ursachen von Beschwerden nach Cholecy stektomie (285) 77 Lessen, H. van und Hupe, K. (Marburg): Leberschäden bei Gallensteinleiden als Ursache eines Postcholecystektomie-Syndroms (290) 82 
Dalichau, H. und Ungeheuer, E. (Frankfurt): Das «Postcholecystektomiesyndrom» im Lichte der intraoperativen Diagnostik des Gallensystems (293) 85

Vahala, Z. und Charvát, A. (Prag): Sekundäre Sphinkterotomie bei der Behandlung des postcholecystektomischen Syndroms $\quad$ (296) 88

Matakas, F. (Köln): Symptomatik und Therapie bei Gallenblasenoperierten (299) 91

Holomáñ, K. (Bratislava): Zur Pathogenese und Prävention der Krankheitszustände nach der Cholecystektomie (303) 95

Rundtischgespräch zum Thema Colitis ulcerosa

Messmer, K.; Devens, K.; Brendel, W.; Kraemer, M.; Rosenthal, L. und Schwabe, P. (München): Experimentelle Untersuchungen über die Reaktivität der Leberdurchblutung (307) 99

Rinecker, H. und Reíchel, K. (München): Experimentelle Unter suchungen zur Motilität verschiedener Darmabschnitte (311) 103 Steíner, H. und Prenner, P. (Salzburg): Probleme der totalen Dünndarmresektion (315) 107

4. Hauptthema: Stoffwechsel

Müller, F. und Hendrik, A. (Greifswald): Untersuchungen über den sogenannten Curare-Diabetes (318) 110

Cerlek, S. und Urbanke, A. (Zagreb): Wie oft und welche Art von Veränderungen finden wir am Leberparenchym bei Diabetes mellitus ? (322) 114

König, K. und Hölzer, K. H. (Hamburg): Permeabilitätsuntersuchungen an der Leberoberfläche (326) 118

Dölle, W. und Strohmeye $\tau$, G. (Marburg): Die Bedeutung des pH für die arteriovenöse und die Blut-Liquor-Differenz des Ammoniaks bei Leberkranken (330) 122

Matzander, U. und Muting, D. (Homburg): Zur Frage der Entgiftungsleistung der Leber nach porto-cavalen Anastomosen (334) 126 Juchems, R. und Gross, W. (Würzburg): Zur Wirkung von Cholinphosphatiden auf die Elektrolytausscheidung bei histologisch gesicherten Fettlebern (340) 132

Klein, U. E. (Kiel): Zur Organspezifität multipler alkalischer Phosphatasen des Serums (345) 137

213 Tagung Inhaltsverzeichnis Suppl. 5 Gross, W.; Hausamen, T.-U. und Rick, W. (Frankfurt): Zur Bestimmung der Aktivität und Spezifität der alkalischen Serumphosphatase (348) 140

Hermanek, P. (Wien): Pilzvorkommen in der Gallenblase （352) 144 5. Hauptthema: Funktionsdíagnostík des Magens Heínkel, K. (Bad Windsheim): Die moderne Funktionsdiagnostik des Magens einschliesslich der Untersuchung mit der Endoradiosonde (355) 147 Weímann, G. (Giessen): Zur Beurteilung der aktuellen Azidität und der Säurebildungsleistung der Magenschleimhaut durch intragastrale pH-Messungen mit der Endoradiosonde («Heidelberger Kapsel») (362) 154 
Himmel, A.; Plonka, A.; Tríef, H. und Wojcíechowska, E. (L·ódz):

Sekretionsgeschwindigkeiten einiger Mineralien des [Magensaftes (365) 157 Mayer, G. und Knauff, H. G. (Marburg): Eine neue Aminosäurelösung zur parenteralen Infusion (369) 161

Kuschke, H. J. und Klusmann, H. (Würzburg): Über eine Freisetzung sympathischer Überträgerstoffe durch Glukagen beim Menschen (373) 165

Drube, H. C. und Hansen, H. T. (Kiel): Untersuchungen über die

Resorption verschiedener Nahrungsfette beim Malabsorptions-

syndrom (376) 168

Bojanowicz, K. (L·ódz): Experimentelle Grundlagen und Regeln der

Behandlung der nicht komplizierten Ulcuskrankheit mit «kalium-

armer Diät» (380) 172

Thorban, W. und Bayíndír, W. (Giessen): Früh- und Spätergebnisse

beim Cardiospasmus nach Behandlung mit der Starckschen Sonde (384) 176

Stiller, H. (Hanau): Die transduodenale Divertikelplastik zur Behand

lung von Duodenaldivertikeln im Papillenbereich (386) 178

Sistek, V.; Rychterova, V. und Horacek, F. (Praha): Barretts Oesophagusulcus (392) 184

Jarosek, H. (Prag): Endoskopische Untersuchungen der Kranken mit Haematemesis und Melaene in akuten Stadien der Blutung -

1Ojährige Erfahrungen (395) 187

Spath, F. und Kronberger, L. (Graz): Die operative Korrektur schlecht

funktionierender Resektionsmägen (postalimentäres Frühsyndrom) (398) 190

Pírker, E. und Kronberger, L. (Graz): Röntgenkinematographische

Funktionsdiagnostik des operierten Magens (402) 194

Sailer, F. X. und Schönbach, G. (Giessen): Die Wirkung der Galle auf

die Durchblutung des Pankreas (405) 197 